\title{
Pacific
}

Journal of

Mathematics

EIGENVALUE BOUNDS AND GIRTHS OF GRAPHS OF FINITE, UPPER HALF-PLANES

NANCY JEANNE CELNIKER

Volume $166 \quad$ No. 1

November 1994 


\section{EIGENVALUE BOUNDS AND GIRTHS OF GRAPHS OF FINITE, UPPER HALF-PLANES}

\section{Nancy Celniker}

For each odd prime power $q=p^{r}$ we will investigate $q-2$ different Cayley graphs called finite, upper half planes over $F_{q}$. We define a finite, upper half-plane by

$$
H_{q}=\left\{x+y \sqrt{d} \mid x, y \in F_{q}, y \neq 0\right\}
$$

where $F_{q}$ is the finite field with $q$ elements and $\mathrm{d}$ is not a square in $F_{q}$. We define a distance $\mathrm{k}$ between points $\mathrm{z}$ and $\mathbf{w} \in H_{q}$ by

$$
k(z, w)=\frac{N(z-w)}{(\operatorname{Im} z)(\operatorname{Im} w)}
$$

where $N z=z \bar{z}$ and $\bar{z}=x-y \sqrt{d}$ and $\operatorname{Re} z=x$ and $\operatorname{Im} z=y$.

We define a graph, $X_{q}(d, a)$, by letting the elements of $H_{q}$ be the vertices of the graph and defining an edge between $\mathbf{z}$ and $\mathbf{w}$ where $k(z, w)=a$ for a fixed $a \in F_{q}-\{0\}$. We consider the origin to be the point $\sqrt{d}$. We call $H_{q}(d, a)$, the finite upper half-plane depending on a fixed $a$ and $d$. We first concern ourselves with whether the eigenvalues, $\lambda$, of the adjacency matrices of the graphs satisfy the Ramanujan bound $|\lambda| \leq \sqrt{q}$. Since the graphs are of degree $q+1$, the paper shows a method to use the representations for the additive and multiplicative groups of each $F_{q}$ to find the smaller associated isospectral matrices. We then find the eigenvalues of the isospectral matrices. A computer program has verified the Ramanujan bound for most of the graphs up to the prime power $3^{5}$. We next concern ourselves with the girth of the graphs. This paper shows that the girths are either 3 or 4 and shows that the girth is 3 if $a=2 d$ and $q \equiv 3(\bmod 4)$ or if $a$ and $a-3 d$ are squares in $F_{q}$. The girth is 4 if $a=2 d$ and $q \equiv 1(\bmod 4)$.

Nicholas M Katz [7] has proven that the eigenvalues of the graphs do satisfy the Ramanujan bound in the paper "Estimates for SotoAndrade Sums-1". Graphs whose eigenvalues satisfy the Ramanujan 
bound are of interest in communications theory as are graphs with certain girths. See Lubotsky [9], Bien [2], Klawe [8] and Chung [5] for applications and more discussion. These graphs are discussed in Terras [12], Celniker et al [4], Poulos [11] and Angel [1].

1. Preliminary Results. Graphs of finite, upper half planes have been defined similarly in other papers. [4], [13]. The graphs in those papers were defined by vertices from the set

$$
H_{p}=\left\{x+y \sqrt{d} \mid x, y \in F_{p}, y \neq 0\right\}
$$

with an analogous definition for the distance between vertices. The initial results about the graphs described here are analogous to the previous results and are included for the reader's convenience.

LEMMA 1.1 (TERras [12]).

(1) The points $z=x+y \sqrt{d}$ and $\sqrt{d}$ are adjacent in

$$
X_{q}(d, a) \Leftrightarrow x^{2}=a y+d(y-1)^{2} .
$$

(2) $X_{q}$ is a regular graph of degree $q+1$ provided that $d$ is a non-square in $F_{q}$ and $a \neq 0$ or $4 d$.

Proof.

(1) Clear from the proof of (2).

(2) We can rewrite the equation in part 1 of the lemma as a norm equation in the finite field $F_{q}(\sqrt{d}): N(z+c)=n$, where $z=x+y \sqrt{d}, c=\sqrt{d}\left(\frac{a}{2 d}-1\right)$, and $n=\frac{a(4 d-a)}{4 d}$. But now the norm in $F_{q}(\sqrt{d})$ is given by $\mathrm{Nz}=z \bar{z}=z z^{q}=z^{q+1}$. Therefore we are solving the equation $w^{q+1}=\mathrm{n}$, for $\mathrm{w}=\mathrm{z}+\mathrm{c}$. Since we are in a finite field, there are exactly $q+1$ solutions as long as $r \neq 0$, which is the case when $a \neq 4 d$.

We notice we can find all the different $X_{q}(d, a)$ by fixing $\mathrm{d}$ and letting $a \in F_{q}-\{0\}, a \neq 4 d$.

From now on we will write $H_{q}(d, a)$ to denote the finite upper half-plane depending on a fixed $d$ and $a$ as defined earlier. 
We recall the definition of the affine group,

$$
\operatorname{Aff}(2, q)=\left\{\left(\begin{array}{ll}
y & x \\
0 & 1
\end{array}\right) \mid x, y \in F_{q}, y \neq 0\right\} .
$$

So we associate the vertices of $X_{q}(d, a)$ with the elements of $\operatorname{Aff}(2, q)$. We will denote elements of $\operatorname{Aff}(2, q)$ by $(y x)$. We now define

$$
S_{q}(d, a)=\left\{x+y \sqrt{d} \in \operatorname{Aff}(2, q): x^{2}=a y=d(y-1)^{2}\right\} .
$$

The affine group acts on $z \in H_{q}$ by the linear transformation

$$
g z=a z+b \in H_{q}, g \in \operatorname{Aff}(2, q) .
$$

We notice that $k(z, w)=k(g z, g w)$ since

$$
\begin{gathered}
N(g z-g w)=N(a(z-w))=N(a) N(z-w)=a^{2} N(z-w) \\
\text { and } \operatorname{Im}(g z)=a \operatorname{Im}(z) .
\end{gathered}
$$

Theorem 1.2 (ANgel [1] ANd Poulos [11]). For all odd primes $p, S_{q}(d, a)$ generates $\operatorname{Aff}(2, q)$.

Proof. From part (2) of Lemma 1.1, we know there are $q+1$ elements of the set $S_{q}$ defined by the equation in part 1. Since $y \neq$ 0 , there must be at least one $(y x) \in S_{q}$ with $x \neq 0$. Since $S_{q}$ is symmetric, for $(y x) \in S_{q},(\mathrm{y}-\mathrm{x}),\left(y^{-1} y^{-1} \mathrm{x}\right)$ must also lie in $S_{q}$. Thus $(y x)\left(y^{-1} y^{-1} x\right)=\left(\begin{array}{lll}1 & 2 x\end{array}\right) \in G$. Then $(12 x)^{n}=\left(\begin{array}{ll}1 & 2 n x\end{array}\right) \in G$ for all integers $n$.

The element $(1 b), b \neq 0$, has order $p$, since by induction it can be shown that the $n$th power of $(1 b)$ is $(1 n b)$. Next, the order of the element ( $a b)$ where $a \neq 1$ is the same as the order of $a$. If $b=0$, then it is obvious this is true. If $b \neq 0$, then conjugate $(a b)$ by the element $\left(b^{-1}\left(1-a^{-1}\right) a^{-1}\right)$ and the resulting element is $(a 0)$. Since conjugate elements have the same order, the order of $(a b)$ is the same as the order of $a$.

Next, we see that $\operatorname{Aff}(2, q)$ can be generated by any element of order $p$ and any element of order $q-1$.

Now any element of order $p$ must be of the form $\left(\begin{array}{ll}1 & b\end{array}\right)$. Let $(a$ $b)$ be an element of order $p$. If $a \neq 1$, then the order of $(a b)$ is 
the same as the order of $a$ in the field of $q$ elements. This order must divide $q-1$, and so can't be $p$, as $p$ doesn't divide $q-1$. So $a$ must be 1 , and any element of order $p$ has the form $(1 b)$, where $b \neq 0$. And any element of order $q-1$ has the form $(a c)$ where the order of $a$ is $q-1$. Let $H$ be the subgroup generated by these two elements. Notice that $(a c)(1 b)(a c)^{-1}=(1 a b)$, so repetition of this conjugation will give us elements of the form $\left(1\left(a^{n}\right) b\right)$ for all powers of $n$. Each one of these elements is of order $p$, and we have $q-1$ of them since the order of $a$ is $q-1$. Now, since the order of $\operatorname{Aff}(2, q)$ is $q(q-1)$, Sylow theory tells us that $\operatorname{Aff}(2, q)$ has one $p$-Sylow subgroup of order $q$, containing every element of order $p$. Since $H$ contains every element of order $p$ (there are at most $q-1$ of them, and $H$ contains $q-1$ of them), $H$ contains the $p$-Sylow subgroup. So, $H$ contains a subgroup of order $q$, and as $H$ contains an element of order $q-1, H$ contains a subgroup of order $q-1$. Since $q$ and $q-1$ are relatively prime, the product of these two subgroups will have order $q(q-1)$, and so we must have $H=\operatorname{Aff}(2, q)$. Now, to show that the graphs are connected, we need to show that the set $S_{q}(d, a)$ generates $\operatorname{Aff}(2, q)$. From the above, it is sufficient to show that $S=S_{q}(d, a)$ can generate an element of order $p$ and of order $q-1$. Let $g$ be a generator of $F_{q}$. Let $T=\{y \mid(y x) \in S\}$. Then $\forall y \in T, y=g^{a_{2}}$ for some $i$. Let $c$ be the gcd of all the $a_{\imath}$. Since for each distinct $y \in T$, there are at most two $x$ s.t. $(y x) \in S$, then $T$ contains at most $\frac{2(q-1)}{c}$ elements, as there are at most $\frac{(q-1)}{c}$ elements of the form $\left(g^{a}\right)$ where $c$ divides $a$, and $a$ is between 0 and $q-2$. Since the cardinality of $T$ is $q+1$ including repetitions, we must have $c=1$. Hence the subgroup generated by $S$ contains an element of order $q-1$.

Since $S$ has cardinality $q+1$ and the multiplicative group of $F_{q}$ has order $q-1$, there is some element in $T$, y, s.t. $\left(y x_{1}\right),\left(y x_{2}\right) \in S$, with $x_{1} \neq x_{2}$. Take any other $(c d) \in S$. Consider the element

$$
(c d)\left(y x_{1}\right)(c d)^{-1}\left(y x_{1}\right)^{-1}=\left(1 x_{1}(c-1)+d(1-y)\right) .
$$

If $x_{1}(c-1)+d(1-y) \neq 0$ (which can only happen if $x_{1} \neq(y-1) d *$ $\left.(c-1)^{-1}\right)$, we are done as this element has order $p$. If this is zero, then $x_{2}$ is not $x_{1}$, so doing the same with $\left(y x_{2}\right)$ instead of $\left(y x_{1}\right)$ will give us an element of order $p$. So, the subgroup generated by $S$ 
has an element of order $p$ and an element of order $q-1$, and thus $S$ generates $\operatorname{Aff}(2, q)$.

THEOREM 1.3. The graphs $X_{q}(d, a)$ are Cayley graphs.

Proof. We think of the elements of $\operatorname{Aff}(2, q)$ as being the vertices and $S_{q}(d, a)$ as being the symmetric set or rather that

$$
\left(\begin{array}{ll}
y & x \\
0 & 1
\end{array}\right) \in S_{q}(d, a) \Leftrightarrow\left(\begin{array}{ll}
y & x \\
0 & 1
\end{array}\right)^{-1} \in S_{q}(d, a) .
$$

This is because

$$
\left(\begin{array}{ll}
y & x \\
0 & 1
\end{array}\right)^{-1}=\left(\begin{array}{cc}
1 / y & -x / y \\
0 & 1
\end{array}\right)
$$

and $x^{2}=$ ay $+\mathrm{d} y-1^{2}$ is equivalent to $(-x / y)^{2}=a(1 / y)+$ $d(1 / y-1)^{2}$. Consider $w, z \in \operatorname{Aff}(2, q)$. We need to show

$$
(w, z) \in E \Gamma \Rightarrow w=z s \text { where } s \in S_{q}(d, a) .
$$

$(\sqrt{d} r, \sqrt{d})=(r, \sqrt{d}) \in E \Gamma$. We prove the statement by recalling that $k(z, w)=k(g z, g w)$ and letting $g=z^{-1}$. Then $(g z, g w) \in E \Gamma$ and $z^{-1} w \in S_{q}(d, a)$.

2. Are these graphs good expanders? We now try to determine if the graphs are good expander graphs as graphs of this type are of interest in communications theory. See Lubotsky [9], Bien [2], Klawe [8] and Chung [5] for applications and more discussion. Lubotsky, Phillips and Sarnak [9] show that a regular graph of degree $\mathrm{k}$ is a good expander if it satisfies the Ramanujan bound, meaning that the second largest eigenvalue of the adjacency matrix, $\lambda \leq \sqrt{k-1}$. So we need to find the eigenvalues of the adjacency matrix. Unfortunately the adjacency matrices are of degree $q^{2}-1$, which makes it difficult to find the eigenvalues even on a computer. So we will use representation theory and take a Fourier transform to find eigenvalues of isospectral matrices of smaller dimension that the adjacency matrices. 
3. Representation Theory for the Prime Power Case. We wish to mimic what Terras [12] did in the prime case. However we are not dealing with a cyclic additive group so the representation theory in this case involves taking traces. Also as all the elements of $F_{q}$ are not integers, we cannot talk about taking powers of matrices for arbitrary elements. Thus most of the material in this section is unique to the prime power case. However the definitions in this section allow us to use the same proof in Section 4 that Terras [12] uses in the prime case. Some elementary proofs about traces will be given to help the reader unfamiliar with this material. For information on representation theory, we refer the reader to Diaconis [6], Mackey [10], and Terras [13].

Since $F_{q}-\{0\}$ is a cyclic multiplicative group with primitive element $g$, we can find the one-dimensional eigenvalues in a similar way to the prime case. Let

$$
\chi_{\jmath}(y)=\exp \left(\frac{(2 \pi i j k)}{q-1}\right), \quad \text { if } \quad y=g^{k}
$$

where $0 \leq \mathrm{j} \leq q-1$.

This leads to a representation we will also call $\chi_{j}$ :

$$
\chi_{j}\left(\begin{array}{ll}
y & x \\
0 & 1
\end{array}\right)=\chi_{j}(y) .
$$

For the $q$-1 dimensional eigenvalues, we would like to define the representation as we did earlier but we notice that $F_{q}$ is not a cyclic additive group. We think of it as the cross product $F_{q}=F_{p} \times F_{p} \times$ $\ldots \times F_{p} \mathrm{r}$ times. So we need to consider the Galois group of order $\mathrm{r}$.

Let $\sigma(x)=x^{p}$. Then $\operatorname{Tr}(\mathrm{x})=\sum_{i=0}^{n-1} \sigma^{\imath}(x)$.

LEMma 3.1. $\operatorname{Tr}(x+y)=\operatorname{Tr}(x)+\operatorname{Tr}(y)$.

Lemma 3.2. For $p$ odd, $\operatorname{Tr}(g(-x))=-\operatorname{Tr}(g x)$.

Then as in the prime case, we can define the additive character of $F_{p}$,

$$
\Psi(x)=\exp \left(\frac{(2 \pi i x)}{p}\right), \quad \text { for } \mathrm{x} \in F_{p}
$$


and define

$$
\tilde{\Psi}(x)=\Psi(\operatorname{Tr}(x))
$$

LEMMA 3.3.

$$
\tilde{\Psi}(u+v)=\tilde{\Psi}(u) \tilde{\Psi}(v)
$$

We need a representation for the additive group of $F_{q}$. We will use the abbreviation $(\mathrm{y} x)=\left(\begin{array}{ll}y & x \\ 0 & 1\end{array}\right)$. Then we define the induced representation, $\rho=\operatorname{Ind} d_{N}^{G} \tilde{\Psi}$,

$$
\begin{gathered}
\text { where } \mathrm{N}=\left\{\left(\begin{array}{ll}
1 & b \\
0 & 1
\end{array}\right): b \in F_{q}\right\} \\
\text { and } \mathrm{G}=\text { Affine group }=\left\{\left(\begin{array}{ll}
y & x \\
0 & 1
\end{array}\right): y \neq 0, x, y \in F_{q}\right\} .
\end{gathered}
$$

$\Psi$ gives a 1-dimensional representation of $\mathrm{N}$ via

$$
\tilde{\Psi}\left(\begin{array}{ll}
1 & b \\
0 & 1
\end{array}\right)=\tilde{\Psi}(b) .
$$

The matrix $\rho(\mathrm{t}), \mathrm{t} \in \mathrm{G}$, is obtained from right translation of functions in the space

$$
\begin{gathered}
V_{\tilde{\Psi}}=\{f: G \rightarrow \mathcal{C} \mid f(n x)=\tilde{\Psi}(n) f(x) \text { for all } n \in \mathcal{N} \text { and all } x \in G\} \\
\text { Define }(\rho(g) f)(x)=f(x g) \forall x, g \in G .
\end{gathered}
$$

This is a group representation since

$$
\begin{aligned}
\left(\rho\left(g_{1} g_{2}\right) f\right)(x) & =f\left(x g_{1} g_{2}\right) \\
\left(\rho\left(g_{1}\right) \rho\left(g_{2}\right) f\right)(x) & =\left(\rho\left(g_{2}\right) f\right)\left(x g_{1}\right) \\
& =f\left(x g_{1} g_{2}\right) .
\end{aligned}
$$

Choose the basis of $V_{\tilde{\Psi}}$ given by

$$
f_{j}(\mathrm{y} \mathrm{x})=\tilde{\Psi}(x) \delta(\mathrm{i} \mathrm{j}) \quad \text { if } \quad y=g^{i}, 0 \leq q-2 .
$$

And define $\delta(i, j)=1$ if $\mathrm{i}=\mathrm{j}$ and 0 otherwise.

We now notice that $(\mathrm{a} b)=\left(\begin{array}{ll}1 & b\end{array}\right)\left(\begin{array}{ll}a & 0\end{array}\right)$. Since representations are homomorphisms, if we wish to see what happens to an element 
(a b) $\in \operatorname{Aff}(2, q)$ we can consider what happens to (a 0$)$ under the induced representation and what happens to $(1 \mathrm{~b})$ under the induced representation. So $\rho\left(\begin{array}{ll}a & b\end{array}\right)=\rho\left(\begin{array}{ll}1 & b\end{array}\right) \rho\left(\begin{array}{ll}a & 0\end{array}\right)$. Then $\left(\rho\left(\begin{array}{ll}1 & b\end{array}\right) f_{j}\right)\left(\begin{array}{ll}y & x\end{array}\right)=$ $f_{j}\left(\left(\begin{array}{ll}y & x\end{array}\right)\left(\begin{array}{ll}1 & b\end{array}\right)\right)$. And $\left(\rho\left(\begin{array}{ll}a & 0\end{array}\right) f_{j}\right)(y x)=f_{j}\left(\left(\begin{array}{ll}y & x\end{array}\right)\left(\begin{array}{ll}a & 0\end{array}\right)\right)$.

LEMMA 3.4.

$$
\left(\rho(1 \quad b) f_{j}\right)(y x)=\tilde{\psi}\left(g^{j} b\right) f_{j}(y x)
$$

Proof. We use Lemma 3.2, the fact that $y=g^{i}$ for $g$ a primitive root and the fact that $\delta(i, j)=0$ for $i \neq j$.

$$
\begin{aligned}
\left(\rho(1 b) f_{j}\right)(y x) & =f_{j}((y x)(1 b))=f_{j}(y y b+x) \\
& =f_{j}\left(g^{i} g^{i} b+x\right)=\tilde{\psi}\left(g^{i} b+x\right) \delta(i, j) \\
& =\tilde{\psi}\left(g^{i} b\right) \tilde{\psi}(x) \delta(i, j)=\tilde{\psi}\left(g^{j} b\right) \tilde{\psi}(x) \delta(i, j) \\
& =\tilde{\psi}\left(g^{j} b\right) f_{j}(y x) .
\end{aligned}
$$

LEMMA 3.5.

$$
\left(\rho(a 0) f_{j}\right)(y x)=\tilde{\psi}(x) \delta(t+i, j)
$$

Proof. We use that $a=g^{t}, y=g^{i}$.

$$
\begin{aligned}
\left(\rho(a 0) f_{j}\right)(y x) & =f_{j}((y x)(a 0))=f_{j}(y a x) \\
& =f_{j}\left(g^{t} g^{i} x\right)=f_{j}\left(g^{t+i} x\right)=\tilde{\psi}(x) \delta(t+i, j) .
\end{aligned}
$$

LEMмA 3.6. For $g=(a b) \in A f f(2, q), \rho(g)=D W^{\log a}$

$$
\text { where } D=\left(\begin{array}{cccc}
\tilde{\Psi}\left(g^{0} b\right) & 0 & 0 \ldots & 0 \\
0 & \tilde{\Psi}\left(g^{1} b\right) & 0 \ldots & 0 \\
\vdots & 0 & 0 & \ddots \\
0 & 0 & 0 \ldots \tilde{\Psi}\left(g^{q-2} b\right)
\end{array}\right)
$$




$$
\text { and where } W=\left(\begin{array}{ccccc}
0 & 1 & 0 & \ldots & 0 \\
0 & 0 & 1 & \ldots & 0 \\
\vdots & 0 & 0 & \ddots & 0 \\
0 & 0 & 0 & \ldots & 0 \\
1 & 0 & 0 & \ldots & 0
\end{array}\right)
$$

Proof. We have already determined that $\rho\left(\begin{array}{ll}a & b\end{array}\right)=\rho\left(\begin{array}{lll}1 & b\end{array}\right) \rho\left(\begin{array}{ll}a & 0\end{array}\right)$. So we see the representation is a product of two matrices which we can call D and U. By Lemma 3.4 we get that

$$
D_{i, j}= \begin{cases}\tilde{\psi}\left(g^{j} b\right) & \text { if } \mathrm{i}=\mathrm{j} \\ 0 & \text { otherwise. }\end{cases}
$$

We see that $\mathrm{D}$ is a diagonal matrix with jth entry $\tilde{\psi}\left(g^{j} b\right)$. By Lemma 3.5 we get that

$$
U_{i, j}= \begin{cases}1 & \text { if } \log a+i=j \\ 0 & \text { otherwise }\end{cases}
$$

We see that $U$ is an $\log a$-shift from the identity matrix and thus $U=W^{\log a}$.

4. Fourier Transform. To mimic the prime case as much as we can, we apply the Fourier transform to the adjacency operator which we define to be

$$
\mathcal{A} f(x)=\sum_{\mathrm{y} \text { adjacent to } \mathrm{x}} f(y)
$$

We then see that

$$
\mathcal{A} f(x)=\sum_{s \in S} f(s x)
$$

because $\mathrm{y}=\mathrm{sx}$ is true for $s \in S$ as shown earlier. We recall the definition of the Fourier transform $\mathcal{F}: L^{2}(G) \mapsto L^{2}(\hat{G})$ by

$$
\mathcal{F} f(\rho)=\hat{f}(\rho)=\sum_{g \in G} f(g) \rho(g) .
$$


We define convolution between $\mathrm{f}$ and $\mathrm{g}$ to be

$$
f * g(x)=\sum_{y \in G} f(y) g\left(y^{-1} x\right) \text {. }
$$

It is known that

$$
\mathcal{F}(f * g)(x)=\mathcal{F}(f(x)) \mathcal{F}(g(x)) .
$$

THEOREM 4.1. Let $A$ be the adjacency matrix of $H_{q}$. Now, as in the prime case if we take the Fourier Transform to diagonalize the adjacency matrix, we get

$$
\mathcal{F} A \mathcal{F}^{-1}=\left(\begin{array}{cc}
R & 0 \\
0 & \tilde{M}
\end{array}\right)
$$

where $R$ is a $q \times q$ diagonal matrix with diagonal entries $R_{j}$ coming from the one-dimensional representations of the Affine Group. (Thus the eigenvalue $q+1$ would occur here as it occurs only once.) Then

$$
R_{j}=\sum_{(x, y) \in S_{q}(d, a)} \chi_{j}(y)
$$

for $\chi_{j}(y)$ defined earlier and $O \leq j \leq q-2$. Then $\tilde{M}$ is a block diagonal matrix with $(q-1)$ identical blocks down the diagonal where each block is

$$
M=\sum \rho(s)=\sum_{(x, y) \in S_{q}(d, a)} D W^{\log y}
$$

where $\rho$ is the $q-1$ dimensional representation of $\operatorname{Aff}(2, q)$ over $F_{q}$ and $D$ and $W$ are as defined in Lemma 3.6.

Proof. We will mimic Terras's [12] proof of the prime case.

We define for $x \in G$

$$
1_{S}(x)= \begin{cases}1 & \text { if } x \in S \\ 0 & \text { otherwise. }\end{cases}
$$

Then we notice that

$$
\begin{aligned}
\mathcal{A} f(x) & =\sum_{s \in S} f(s x)=\sum_{y \in G} 1_{S}(y) f\left(y^{-1} x\right) \\
& =1_{S} * f(x) .
\end{aligned}
$$


Then

$$
\begin{aligned}
\mathcal{F} A \mathcal{F}^{-1} k \rho & =\mathcal{F}\left(A \mathcal{F}^{-1} k\right) \rho=\mathcal{F}\left(1_{S} * \mathcal{F}^{-1} k\right) \rho \\
& =\left(\mathcal{F} 1_{S} \rho\right)\left(\mathcal{F} \mathcal{F}^{-1} k \rho\right)=\left(\sum_{g \in \operatorname{Aff}(2, q)} 1_{S}(g) \rho(g)\right) k \rho .
\end{aligned}
$$

If we choose first for $\mathrm{k}$ to be those elements of the basis of $L^{2}(\hat{G})$ which correspond to the one-dimensional representations of $\operatorname{Aff}(2, q)$, then we see we get a diagonal matrix with jth entry of $\chi_{j}(y)$.

If we choose next for $\mathrm{k}$ those elements of the basis of $L^{2}(\hat{G})$ which correspond to the $q$ - 1 dimensional representation of $\operatorname{Aff}(q)$. We then notice that the basis elements look like

$$
(x, 0, \ldots, 0),(0, x, \ldots, 0), \ldots,(0, \ldots, 0, x) .
$$

Then we see that $M X=(M x, M x, M x, \ldots, M x)$ if we look at it in columns. Thus this corresponds to $q-1$ copies of the $M$ matrix running diagonally down.

5. Graphs with similar spectra in the prime power case. We will now talk about different graphs which have similar spectra. We will denote the two graphs $X_{q}\left(d, a_{1}\right)$ and $X_{q}\left(d, a_{2}\right)$ since the graphs will be over the same field, use the same non-square and will differ only in the " $a$-distance" between vertices. Then the corresponding $M$ submatrices described in Theorem 4.1 will be called $M_{1}$ and $M_{2}$. And the matrix $W$ is as defined in Lemma 3.6.

Proposition 5.1. If $a_{1}+a_{2} \equiv 4 d(\bmod q)$ then $M_{2}=M_{1} W^{\frac{q-1}{2}}$.

Proof.

$$
\begin{aligned}
a_{1}+a_{2} \equiv 4 d(\bmod q) & \Leftrightarrow \\
y_{1}\left(a_{1}+a_{2}\right) \equiv 4 y_{1} d(\bmod q) & \Leftrightarrow \\
a_{1} y_{1}+d y_{1}{ }^{2}-2 d y_{1}+d \equiv-a_{2} y_{1}+d y_{1}{ }^{2}+2 d y_{1}+d(\bmod q) & \Leftrightarrow \\
a_{1} y_{1}+d\left(y_{1}-1\right)^{2} \equiv-a_{2} y_{1}+d\left(-y_{1}-1\right)^{2}(\bmod q) & \Leftrightarrow \\
x_{1}{ }^{2} \equiv x_{2}{ }^{2} \text { and } y_{1} \equiv-y_{2} & \Leftrightarrow \\
\left(x_{1}, y_{1}\right) \in S_{q}\left(a_{1}, d\right) & \Leftrightarrow \\
\left(x_{1},-y_{1}\right) \in S_{q}\left(a_{2}, d\right) . &
\end{aligned}
$$




$$
\begin{gathered}
\text { Then } M_{1}=\sum_{(x, y)} D^{x} W^{\log y} \\
M_{2}=\sum_{(x,-y)} D^{x} W^{\log (-y)}=\sum_{(x, y)} D^{x} W^{\log y+\frac{q-1}{2}} \\
=\sum_{(x, y)} D^{x} W^{\log y} W^{\frac{q-1}{2}}=M_{1} W^{\frac{q-1}{2}} .
\end{gathered}
$$

Since $W$ is a 1 -shift from the identity matrix, we see that

$$
W^{\frac{q-1}{2}}=\left(\begin{array}{ll}
0 & I \\
I & 0
\end{array}\right) \text {. }
$$

Proposition 5.2. The $M$ 's are block symmetric matrices.

Proof. Recall

$$
M=\sum \rho(s)=\sum_{(x, y) \in S_{q, a}} D W^{\log y}
$$

and

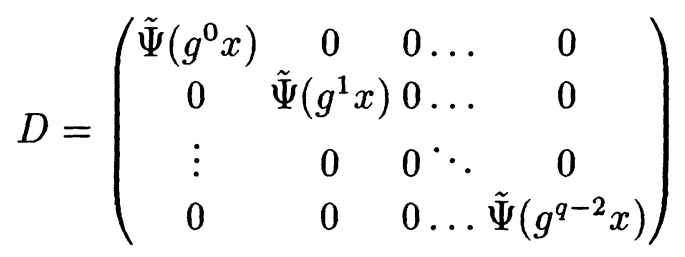

where

$$
\tilde{\Psi}(x)=\exp \left(\frac{(2 \pi i \operatorname{Tr}(x))}{p}\right), \text { for } \mathrm{x} \in F_{p} .
$$

Since the $D$ 's are diagonal and for every $y$ term we get both an $x$ and an $-x$ term the diagonal terms in the sum will be

$$
\begin{aligned}
& \exp \left(\frac{2 \pi i \operatorname{Tr}\left(g^{j} x\right)}{p}\right)+\exp \left(\frac{2 \pi i \operatorname{Tr}\left(g^{j}(-x)\right)}{p}\right) \\
= & \exp \left(\frac{2 \pi i \operatorname{Tr}\left(g^{j} x\right)}{p}\right)+\exp \left(\frac{2 \pi i-\operatorname{Tr}\left(g^{j} x\right)}{p}\right) \\
= & 2 \cos \left(\frac{2 \pi \operatorname{Tr}\left(g^{j} x\right)}{p}\right) .
\end{aligned}
$$


Since $g$ is a primitive root of $q$

$$
\begin{aligned}
& \operatorname{Tr}\left(g^{j+\frac{q-1}{2}} x\right)=\operatorname{Tr}\left(g^{j} g^{\frac{q-1}{2}} x\right) \\
= & \operatorname{Tr}\left((-1) g^{j} x\right)=-\operatorname{Tr}\left(g^{j} x\right)
\end{aligned}
$$

and thus

$$
\cos \left(\frac{2 \pi}{p} \operatorname{Tr}\left(g^{j+\frac{p-1}{2}} x\right)\right)=\cos \left(-\frac{2 \pi}{p} \operatorname{Tr}\left(g^{j} x\right)\right)=\cos \left(\frac{2 \pi}{p} \operatorname{Tr}\left(g^{j} x\right)\right)
$$

so we see the sum of the $D$ 's for opposite values of $x$ leads to a block symmetric matrix.

Now we notice that $W$ is block symmetric. In the $(p-1) / 2 \times$ $(p-1) / 2$ submatrix (call it 1$)$ of $W$ we have a single right shift of I followed by a row of the zero matrix. Then the lower $(p-1) / 2 \times(p-$ $1) / 2$ submatrix of $W$ (call it 4 ) is identical as it does not include the row with the 1 entry in the $\frac{p-1}{2}+1$ place but includes all after it. Then the submatrix to the right of 1 contains all zeros except for a 1 in the first column of the (last) $\frac{p-1}{2}$ row. Then the submatrix to the left of 4 contains all zeros except for a 1 in the first column of the last row. So $W$ is block symmetric.

We also see that each $W^{\log y}$ is of block symmetric matrix form as. we can see by induction.

$$
\begin{aligned}
& \text { Let } \mathrm{W}=\left(\begin{array}{ll}
U & V \\
V & U
\end{array}\right) . \\
& \text { Then } W^{2}=\left(\begin{array}{cc}
U^{2}+V^{2} & U V+V U \\
V U+U V & U^{2}+V^{2}
\end{array}\right) \text {. } \\
& \text { Now assume } W^{j}=\left(\begin{array}{ll}
A & B \\
B & A
\end{array}\right) \text {. } \\
& \text { Then } W^{j+1}=W^{j} W=\left(\begin{array}{l}
U A+V B U B+V A \\
V A+U B U A+V B
\end{array}\right) .
\end{aligned}
$$

The previous proof also shows us that $\left(D^{x}+D^{-x}\right) W^{\log y}$ is block symmetric. An extremely simple induction shows us that

$$
\mathrm{M}=\sum_{(x, y)} D^{x} W^{\log y}
$$


is also block symmetric.

Proposition 5.3. If $a_{1}+a_{2} \equiv 4 d(\bmod q)$, then the eigenvalues of the $M$ 's are the same up to a factor of -1 .

Proof. Using Propositions 5.1 and 5.2 we see that

$$
M_{1}=\left(\begin{array}{ll}
A & B \\
B & A
\end{array}\right) \Rightarrow M_{2}=\left(\begin{array}{ll}
B & A \\
A & B
\end{array}\right)
$$

Then we take the Fourier transform to block diagonalize the matrices.

$$
\begin{gathered}
\text { Let } \mathcal{F}=\left(\begin{array}{cc}
I & I \\
-I & I
\end{array}\right) \\
\text { Then } \mathcal{F}^{-1}=\left(\begin{array}{cc}
I & -I \\
I & I
\end{array}\right) \\
\text { So } F M_{1} F^{-1}=\left(\begin{array}{cc}
2(A+B) & 0 \\
0 & 2(A-B)
\end{array}\right) \\
\text { But then } F M_{2} F^{-1}=\left(\begin{array}{cc}
2(B+A) & 0 \\
0 & 2(B-A)
\end{array}\right) .
\end{gathered}
$$

COROLLARY 5.4. If $a=2 d$, the graph has at least $\frac{q-1}{2}$ zero eigenvalues.

6. Computer Implementation. The two major differences in computing this case versus the prime case is that first of all $F_{q}$ unlike $F_{p}$ is not a cyclic additive group. Thus the software for computing the elements in $S_{q}(\mathrm{~d}, \mathrm{a})$ versus $S_{p}(\mathrm{~d}, \mathrm{a})$ and the representations for $\operatorname{Aff}(2, q)$ versus $\operatorname{Aff}(2, p)$ is quite different.

Since the elements of $F_{q}$ are the elements of the splitting field $x^{q}-x=0$, one element is 0 and the others are the $p^{r-1}$ roots of unity. We can think of these elements as powers of the primitive root (a multiplicative group) or as linear combinations of the primitive root over the field $F_{p}$. 
In some cases using the additive properties is better and in other using the multiplicative properties is better so in the computer implementation this time, the program computes a logarithm table for the powers of the primitive root. Then in calculating the solutions to

$$
x^{2}=a y+d(y-1)^{2}
$$

the program switches back and forth in the log table depending on the if the operation is better suited to the additive or multiplicative properties. If $x=0$ is a solution this is a special case as it is not a power of the primitive root and does not belong in the log table. This special case occurs if and only if $(a-4 d) a$ is a square as will be seen in the Lemma 8.3 so the program accounts for this situation.

The program then finds the one-dimensional and $(q-1)$-dimensional eigenvalues as shown in Lemma 5.9 and compare them with the Ramanujan bound. The program uses the multiplicative properties to find the trace and representation as it is easy to take exponents. The program uses the result of Proposition 5.2 to cut the $M$ matrix from being $q-2 \times q-2$ dimensional to $\frac{q-2}{2} \times \frac{q-2}{2}$ dimensional.

7. Computer Results. The program was run on a Solbourne $5 / 804$. This machine is a four CPU system with $64 \mathrm{MB}$ of memory. Each processor is rated at about 13.5 SPEC marks. for a total processing capacity equal to 68 DEC VAX11/780s. The processors implement SUN's SPARC architecture. The operating system software is OS/MP, which is derived from SunOS which is an implementation of UNIX. Computer results show that for $q$ up to $11^{2}$, the Ramanujan bound does hold for all values of a. For values of $a$ up to 42 , the bound holds for $q=13^{2}$. For values of $a$ up to 19 , the bound hold for $q=3^{5}$. The computer account ran out of processor time before more values of $a$ could be checked.

Interestingly, for even powers of the primes 3,5,7,11 and 13, some of the eigenvalues are equal to the Ramanujan bound in the onedimensional and sometimes $q$-dimensional eigenvalues as we see in Table 3. We recall that the one-dimensional eigenvalues from Theorem 4.1 to look like

$$
\sum_{(x, y) \in S_{q}(d, a)} \cos \frac{2 \pi j \log y}{q-1}
$$


because the sin terms will cancel out since sin is an odd function and by an explanation given in Theorem 1.3, both $(y x)$ and $\left(y^{-1}-x y^{-1}\right) \in S_{q}(d, a)$. We now notice that these one-dimensional eigenvalues will be integers for appropriate values of $y$ and $j$ as will each term in the summation. Also for even powers of primes, the Ramanujan bounds will be integers. The Ramanujan bounds are not integers for odd powers of primes.

The table shows the prime power, the $a$ values where the boundary eigenvalues occur, the eigenvalue and the $j$ values as described above and in Theorem 4.1. The $a$ values are given as powers of the primitive root of $F_{q}$ which was used also as $d$.

8. Girths of the $X_{q}$. We now turn to the second purpose of this paper which is to explore the girths of these graphs. We define the girth to be the length of the shortest circuit in a graph. Except for the proof of Theorem 8.4 which shows the girth to be 4 if it is not 3 , the proofs only prove the graphs to have girth 3 .

LEMMA 8.1. The girth of a graph of $H_{q}$ is either 3 or 4.

Proof. We use a result of Tutte's [3] that the number of vertices in a graph of valency $k$ and odd girth is at least

$$
1+k+k(k-1)+\ldots+k(k-1)^{\frac{1}{2}(g-3)} .
$$

So we assume the girth is 5 . Then the number of vertices is at least

$$
n=1+(q+1)+(q+1) q .
$$

This is a contradiction since the number of vertices is $q(q-1)$ and $n>q(q-1)$.

LEMмA 8.2. If the number of origin neighbors who are neighbors is at least one, the girth is 3. Otherwise the girth is 4.

Proof. If $\left(\dot{o}, s_{1}\right),\left(o, s_{j}\right),\left(s_{1}, s_{j}\right) \in E \Gamma$, clearly the girth is 3 . 
TABLE 3. Values of $q, a$ and $j$ For Boundary Eigenvalues

\begin{tabular}{|c|c|c|c|}
\hline$q$ & $\log _{d} a$ & Eigenvalue & j \\
\hline $3^{2}$ & 5 & -6 & 4 \\
\hline $5^{2}$ & 1 & -10 & 12 \\
\hline $5^{2}$ & 7 & -10 & $6,8,16,18$ \\
\hline $5^{2}$ & 9 & -10 & 12 \\
\hline $7^{2}$ & 1 & -14 & 24 \\
\hline $7^{2}$ & 6 & 14 & 16,32 \\
\hline $7^{2}$ & 9 & -14 & 24 \\
\hline $7^{2}$ & 17 & -14 & $8,12,24,36,40$ \\
\hline $7^{2}$ & 36 & 14 & 16,32 \\
\hline $3^{4}$ & 41 & 18 & $10,16,30,32,40,48,50,64,70$ \\
\hline $11^{2}$ & 13 & 22 & 60 \\
\hline $11^{2}$ & 25 & 22 & 40,80 \\
\hline $11^{2}$ & 37 & 22 & $12,20,36,40,60,80,84,100,108$ \\
\hline $11^{2}$ & 53 & 22 & 60 \\
\hline $11^{2}$ & 61 & 22 & 40,80 \\
\hline $11^{2}$ & 85 & -22 & 30,90 \\
\hline $11^{2}$ & 93 & 22 & 60 \\
\hline $11^{2}$ & 97 & -22 & 30,90 \\
\hline $11^{2}$ & 109 & 22 & 60 \\
\hline $13^{2}$ & 15 & 26 & $14,24,42,48,70,96,98,120,126,144,154$ \\
\hline $13^{2}$ & 24 & 26 & 84 \\
\hline $13^{2}$ & 33 & 26 & 84 \\
\hline
\end{tabular}

Now assume $\Gamma$ has girth 3 . Then for some $w_{1}, w_{2}, w_{3} \in V \Gamma$

$$
\left(w_{1}, w_{2}\right),\left(w_{2}, w_{3}\right),\left(w_{3}, w_{1}\right) \in E \Gamma .
$$

Since $k\left(w_{1}, w_{2}\right)=k\left(g w_{1}, g w_{2}\right)$,

$$
\left(g w_{1}, g w_{2}\right) \in E \Gamma,\left(g w_{2}, g w_{3}\right) \in E \Gamma,\left(g w_{3}, g w_{1}\right) \in E \Gamma .
$$

Now letting $g=w_{1}^{-1}$,

$$
\begin{aligned}
& \left(g w_{1}, g w_{2}\right)=\left(o, g w_{2}\right) \Rightarrow g w_{2} \in S \\
& \left(g w_{3}, g w_{1}\right)=\left(g w_{3}, o\right) \Rightarrow g w_{3} \in S .
\end{aligned}
$$

So there is at least one pair of origin neighbors who are neighbors.

LEMMA 8.3.

$$
(y 0) \in S \Leftrightarrow a(a-4 d) \text { is a square in } F_{q} .
$$


Proof.

$$
\begin{aligned}
& 0=a y+d(y-1)^{2} \Leftrightarrow \\
& 0=d y^{2}+(a-2 d) y+d \Leftrightarrow \\
& y=\frac{2 d-a \pm \sqrt{\left.(a-2 d)^{2}-4 d^{2}\right)}}{2 d} \Leftrightarrow .
\end{aligned}
$$

There is a solution for $y \in F_{q} \Leftrightarrow a(a-4 d)$ is a square.

THEOREM 8.4. Let $X_{q}(d, 2 d)=\Gamma$. If $q \equiv 1(\bmod 4)$ the girth of of the graph is 4. If $q \equiv 3(\bmod 4)$, the girth of the graph is 3 .

Proof. Assume $\Gamma$ has girth 3. Then by Lemma 8.2 for any $s_{1} \in S$, there exists $s_{2} \in S$, where $\left(s_{1}, s_{2}\right) \in E \Gamma$.

$$
\begin{gathered}
x_{1}^{2}=a y_{1}+d\left(y_{1}-1\right)^{2}=d y_{1}^{2}+d \\
x_{2}^{2}=a y_{2}+d\left(y_{2}-1\right)^{2}=d y_{2}^{2}+d \\
\frac{\left(x_{2}-x_{1}\right)^{2}-d\left(y_{2}-y_{1}\right)^{2}}{y_{1} y_{2}}=a \Leftrightarrow \\
x_{2}^{2}-2 x_{1} x_{2}+x_{1}^{2}-d y_{2}^{2}+2 d y_{1} y_{2}-d y_{1}^{2}=a y_{1} y_{2} \Leftrightarrow \\
d y_{2}^{2}+d-2 x_{1} x_{2}+d y_{1}^{2}+d-d y_{2}^{2}+2 d y_{1} y_{2}-d y_{1}^{2}=a y_{1} y_{2} \Leftrightarrow \\
2 d-2 x_{1} x_{2}=a y_{1} y_{2}-2 d y_{1} y_{2} \Leftrightarrow \\
2\left(d-x_{1} x_{2}\right)=(a-2 d) y_{1} y_{2} \Leftrightarrow \\
-2 x_{1} x_{2}=-2 d+(a-2 d) y_{1} y_{2} \Leftrightarrow x_{1} x_{2}=d .
\end{gathered}
$$

First we consider the case $q \equiv 1(\bmod 4)$. We notice that -1 is a square. By the above equations, we have

$$
y_{1}^{2}+1=x_{1}^{2} / d=d / x_{2}^{2}
$$

and

$$
y_{2}^{2}+1=x_{2}^{2} / d=d / x_{1}^{2} .
$$

We notice that for $i \in 1,2 y_{i}^{2} \neq-1$ since then $x_{i}^{2}=0$ and $x_{1} x_{2}=0$. Therefore

$$
\left(y_{1}^{2}+1\right)\left(y_{2}^{2}+1\right)=y_{1}^{2} y_{2}^{2}+y_{1}^{2}+y_{2}^{2}+1=y_{1}^{2}\left(y_{2}^{2}+1\right)+y_{2}^{2}+1 \equiv 1 .
$$


But this implies that

$$
y_{2}^{2}+1 \equiv \frac{-y_{2}^{2}}{y_{1}^{2}}
$$

But the right hand is a square, while the left hand is not (since it's equal in $F_{q}$ to $x_{2}^{2} / d$, and $d$ is not a square), so we have a contradiction, and the girth must be 4 .

Now we wish to show that for $q \equiv 3(\bmod 4)$, the girth is 3 . We will show that

$$
\forall\left(\begin{array}{ll}
y_{1} & x_{1}
\end{array}\right) \in S \exists\left(\begin{array}{ll}
y_{2} & x_{2}
\end{array}\right) \in S \quad \text { so } \quad x_{1} x_{2}=d \text {. }
$$

Given $\left(y_{1} x_{1}\right) \in S$. Then

$$
x_{1}^{2}=d y_{1}^{2}+d \Leftrightarrow y_{1}^{2}=\frac{x_{1}^{2}-d}{d} \Leftrightarrow \frac{x_{1}^{2}-d}{d} \quad \text { is a square. }
$$

Recall that $d=g$, the first primitive root in $F_{q}$. We notice that for $q \equiv 3(\bmod 4)$, if $\mathrm{g}$ is the first primitive root in $F_{q}, g^{\frac{q-3}{2}}$ is a square.

$$
g^{\frac{q-3}{2}}=g^{\frac{q-1}{2}-1}=\frac{g^{\frac{q-1}{2}}}{g}=\frac{-1}{d} .
$$

Thus $\frac{-1}{d}$ is a square. Then, $-\frac{x_{1}^{2}}{d}$ is a square, and since we already determined that $\frac{x_{1}^{2}-d}{d}$ is a square,

$$
\frac{x_{1}^{2}-d}{d}=\left(\frac{d}{x_{1}^{2}}-1\right)\left(-\frac{x_{1}^{2}}{d}\right) \Rightarrow
$$

$\frac{d}{x_{1}^{2}}-1$ is a square $\Rightarrow \exists y_{2}$ where $y_{2}^{2}=\frac{d}{x_{1}^{2}}-1$

Thus $y_{2}^{2}+1=\frac{d}{x_{1}^{2}} \Rightarrow d y_{2}^{2}+d=\frac{d^{2}}{x_{1}^{2}} \Rightarrow$

$$
\left(y_{2} \frac{d}{x_{1}}\right)=\left(y_{2} x_{2}\right) \in S
$$


Lemma 8.5. $(1 \sqrt{a}) \in S \Leftrightarrow a$ is a square in $F_{q}$. $a$.

Proof. Since $x^{2}=a y+d(y-1)^{2}, y=1, y=\frac{d-a}{d} \Leftrightarrow x^{2}=$

THEOREM 8.6. In $X_{q}(d, a)$, if $a$ is a square in $F_{q}$ and $a-3 d$ is a square in $F_{q}$ then the girth is 3.

Proof. By Lemma 8.5, if $a$ is a square in $F_{q},(1 \sqrt{a}) \in S$. We see that the neighbors of $s_{i} \in S$ are of the form $s_{i} s_{j}$ for $1 \leq j \leq q+1$ since $s_{i}$ is a neighbor of the origin and we do a left multiplication of the origin neighbors by $s_{i}$ to get the neighbors of $s_{i}$.

$$
(1 \sqrt{a})\left(y_{l} x_{l}\right)=\left(y_{l} x_{l}+\sqrt{a}\right) .
$$

By Lemma $8.2 X_{q}(d, a)$ has girth 3 whenever

$$
\exists\left(y_{l} x_{l}\right) \in S \quad \text { such that } \quad\left(y_{l} x_{l}+\sqrt{a}\right) \in S \text {. }
$$

Since $(y x) \in S \Leftrightarrow x^{2}=a y+d(y-1)^{2}$, and we notice that the right hand side of the equation only depends on $\mathrm{y}$, so both $\left(y_{l} x_{l}\right),\left(y_{l} x_{l}+\right.$ $\sqrt{a}) \in S$, if

$$
x_{l}^{2}=\left(x_{l}+\sqrt{a}\right)^{2} \Leftrightarrow x_{l}=-\frac{\sqrt{a}}{2} .
$$

Then

$$
(1 \sqrt{a})\left(y_{l}-\frac{\sqrt{a}}{2}\right)=\left(y_{l} \frac{\sqrt{a}}{2}\right) .
$$

$$
\begin{gathered}
\text { Now } \quad\left(y_{l} \frac{\sqrt{a}}{2}\right) \in S \Leftrightarrow \\
\frac{a}{4}=a y_{l}+d\left(y_{l}-1\right)^{2} \Leftrightarrow \\
0=d y_{l}^{2}+(a-2 d) y_{l}+\left(d-\frac{a}{4}\right) \Leftrightarrow \\
y_{l}=\frac{(2 d-a) \pm \sqrt{a^{2}-3 a d}}{2 d} .
\end{gathered}
$$

And there is a solution for $y_{l} \Leftrightarrow a^{2}-3 a d$ is a square. 


\section{REFERENCES}

[1] J. Angel, Ph.D. Thesis, (U.C.S.D. 1993).

[2] F. Bien, Construction of Telephone Networks by Group Representations, Notices of the A.M.S., 36 (1989), 5-22.

[3] N. Biggs, Algebraic Graph Theory, Cambridge University Press, London 1974.

[4] N. Celniker, S. Poulos, A. Terras, C. Trimble and E. Velasquez, Is There Life on Finite, Upper Half Planes?, to appear in Contemporary Math., Amer. Math. Soc., 1990.

[5] F. R. K. Chung, Diameters and Eigenvalues, J. of the Amer. Math Soc. , 2 (1980), 187-196.

[6] P. Diaconis, Group Representations in Probability and Statistics, Inst. of Math. Stat., Lect. Note-Monograph Series, Haywood, 11 (1988).

[7] N. Katz, Estimates for Soto-Andrade Sums - 1, pre-print.

[8] M. Klawe, Limitations on explicit constructions of expanding graphs, S.I.A.M. J. Comput. 13 (1984), 156-166.

[9] A. Lubotsky, R. Phillips, and P. Sarnak, Ramanujan Graphs, Combinatorica, 8 (1988), 261-277.

[10] G. Mackey, Unitary Group Representations in Physics, Probability and Number Theory, Benjamin/Cummings, Reading, Mass, 1978.

[11] S. Poulos, Ph.D. Thesis, U.C.S.D. 1991.

[12] A. Terras, Eigenvalue Problems Related to Finite Analogues of Upper Half-Planes, to appear in H. Weyl memorial Volume, Texas A\&M University, 1991.

[13] A. Terras, Harmonic Analysis on Symmetric Spaces and Applications, I,II 1985,1988, Springer-Verlag, New York.

Received June 20, 1991, revised September 11, 1993 and accepted for publication April 11, 1994.

Whittier College

Whittier, CA 90608 




\section{PACIFIC JOURNAL OF MATHEMATICS}

Volume $166 \quad$ No. $1 \quad$ November 1994

Eigenvalue bounds and girths of graphs of finite, upper half-planes

NANCY JEANNE CELNIKER

On the compactness of a class of Riemannian manifolds

ZHIYONG GAO and GUOJUN LiAO

The distribution mod $n$ of fractions with bounded partial quotients

Douglas Austin Hensley

Paired calibrations applied to soap films, immiscible fluids, and

surfaces or networks minimizing other norms

GARY REID LAWLOR and FRANK MORGAN

Conformal repellors with dimension one are Jordan curves

R. DANIEL MAULDIN and MARIUSZ URBANSKI

Order of the identity of the stable summands of $\Omega^{2 k} S^{2 n+1}$

PAUl Silberbush

On a construction of pseudo-Anosov diffeomorphisms by sequences of 123 train tracks

ITARU TAKARAJIMA

On systems of generators of arithmetic subgroups of higher rank groups

T. N. VENKATARAMANA 